\title{
The Effects of Soybean Trypsin Inhibitor on Free Radicals Levels in Pancreatic Mitochondria of Mice
}

\author{
Chunmei GU ${ }^{1}$, Shujun $\mathrm{Li}^{1}$, Linlin $\mathrm{ZHAO}^{1}$, Xinxiu SONG ${ }^{1}$, Guixin $\mathrm{QIN}^{2, *}$ \\ ${ }^{1}$ Institute of Food Science and Engineering, Jilin Agricultural University, Changchun, China \\ ${ }^{2}$ Institute of Animal Science and Technology, Jilin Agricultural University, Changchun, China \\ *Corresponding author: qgx@jlau.edu.cn
}

Received June 20, 2014; Revised July 02, 2014; Accepted July 07, 2014

\begin{abstract}
This paper aims to explore the effect of soybean trypsin inhibitor (STI) on oxygen free radicals levels in pancreatic mitochondria of mice. 36 male KM mice were randomly divided into three groups and fed with different diets as follows: control group was fed on control diet, STI group was fed on control diet containing STI, and VC (vitamin C) group was fed on STI diet supplemented with vitamin C. Mice were sacrificed by decapitation after being fed for 3 weeks. Pancreas was quickly removed and mitochondria were prepared for determining the oxidative and antioxidative index. The results showed that, compared with mice fed with control diet, malondialdehyde (MDA) content and activity of nitric oxide synthase (NOS) in pancreatic mitochondria of mice fed with STI diet were significantly increased $(P<0.05)$, the content of glutathione (GSH), total antioxidant capacity (TAOC), the activities of glutathione peroxidase (GSH-Px), catalase(CAT) and superoxide dismutase (SOD) were significantly dropped. After STI diet was supplemented with antioxidant, the contents of oxidation parameters were significantly decreased $(P<0.05)$, but their values were still higher than those of control group; the activities of antioxidant parameters in pancreatic mitochondria were significantly increased $(P<0.05)$, but still lower than those of control group. These results supported that STI could cause oxidative stress in pancreatic mitochondria. In addition, vitamin C could interfere with the role of STI on the organism and effectively improve the status of oxidative stress.
\end{abstract}

Keywords: soybean trypsin inhibitor, mitochondria, free radical, mice, pancreas

Cite This Article: Chunmei GU, Shujun Li, Linlin ZHAO, Xinxiu SONG, and Guixin QIN, "The Effects of Soybean Trypsin Inhibitor on Free Radicals Levels in Pancreatic Mitochondria of Mice." Journal of Food and Nutrition Research, vol. 2, no. 7 (2014): 357-362. doi: 10.12691/jfnr-2-7-5.

\section{Introduction}

Being a good resource of vegetable protein, soybean is of highly nutritive value. It is rich in a good balance of proteins, amino acids and essential lipids, which confer a good nutritional value. But soybeans contain antinutritional factors which affect the development and utilization of soybean [1]. Early studies found that soybean contains more than ten kinds of anti-nutritional factors [2]. Among them, trypsin inhibitor is the major anti-nutritional factors (ANFs) in soybean seeds [3], and it could result in a serious obstacle when human being consume soybean. It was reported that trypsin inhibitors accounted for $30-50 \%$ of the growth inhibition effect and almost all of the hypertrophic response in the pancreas of animals fed with raw soybean meal. Bau et al. (2001) also discovered that trypsin inhibitor could reduce protease activity and stimulate the synthesis and secretion of the pancreas, which led to hypertrophy and hyperplasia of pancreas [4]. STI also has a negative role under certain circumstances, it will reduce digestibility of nutrients and feed conversion, decrease feed intake and weight, cause mouse and chicken pancreas swelling, and the degree was related with STI levels in the diet $[5,6,7,8]$.
Mitochondria are the main production place of free radicals. It is estimated that approximately $0.2-2 \%$ of the oxygen taken up by a cell is converted by mitochondria to reactive oxygen species (ROS), mainly through the production of superoxide anion radical $\left(\mathrm{O}_{2}{ }^{--}\right)$[9]. It also considered the most equal important cellular organelle to contribute to the aging process, mainly through formation of reactive oxygen species and respiratory chain dysfunction that result in the damage of mitochondrial proteins, lipids, and DNA [10]. Therefore, mitochondria are the main site of cellular biological oxidation and energy conversion. It is not only a major source of ROS, but also the main target of ROS injury. The early studies indicated that STI could do harm to pancreas by increasing the formation of lipid peroxidation [11], but the mechanism remains unclear. So this paper aims to explore the effect of STI on the generation of free radicals in pancreatic mitochondria. Meanwhile, vitamin C (VC) was supplemented to diet in order to further study the change trend of free radicals under the antioxidant intervention.

\section{Material and Methods}

\subsection{Animals and Treatment}


36 male $\mathrm{KM}$ mice were all housed in a temperaturecontrolled $\left(20-22^{\circ} \mathrm{C}\right)$ room with a 12 -h lightedark cycle and had free access to drinking water. After acclimation for 1 weeks, all animals were randomly divided into three groups as follows: control group, STI group and STI+VC group. The mice in control group was fed a control diet. The mice in STI group was fed a control diet containing $4.8 \mathrm{mg} / \mathrm{g}$ STI. The mice in STI+VC group was fed a STI diet supplmented with $1500 \mathrm{mg} / \mathrm{kg}$ Vitamin C. The composition of the control diet was shown in Table 1. All mice were fed for 3weeks. Soybean trypsin inhibitor specific activity was $4600 \mathrm{U} / \mathrm{mg}$. The care and use of the mice followed the institutional guideline of Jilin Agricultural University.

Table 1. Composition of the diets $(\mathrm{g} / \mathrm{kg})^{\text {a }}$

\begin{tabular}{ccc}
\hline & Ingredient & Diet \\
\hline Protein & Casein $^{\mathrm{b}}$ & 200 \\
Carbohydrates & Corn starch $^{\mathrm{b}}$ & 660 \\
Fat & Soybean oil $^{\mathrm{c}}$ & 50 \\
Fibers & Cellulose powder $^{\mathrm{b}}$ & 30 \\
& Mineral mixture $^{\mathrm{d}}$ & 50 \\
Others & Vitamin mixture $^{\mathrm{e}}$ & 10 \\
\hline
\end{tabular}

Note:

a The diets were semipurified, isoenergetic (16.33 MJ/kg).

${ }^{\mathrm{b}}$ Changchun, China.

c The commercial product ( $50 \mathrm{~g} / \mathrm{kg}$ ) provides $11.81 \%$ of energy. The soybean oil provides the following fatty acids: 14:0, traces; C16:0, 10.3; C16:1 $\omega-7,0.1$; C18:0, 3.9; C18:1 $\omega-7+\omega-9,22.1$; C18:2 $\omega-6,54.8$; $\mathrm{C} 18: 3 \omega-3,7.5$; C20:0, 0.4; C20:1 $\omega-9+\omega-11,0.2$; C22:0, 0.4; C22:5 $\omega-$ 3 , traces; C24:0, traces; sum of saturated fatty acids (S), 15; sum of monounsaturated, 22.4; sum of polyunsaturated fatty acids $(\mathrm{P}), 84.7$; $\mathrm{P} / \mathrm{S}$, $5.65 ; \Sigma \omega-6 / \Sigma \omega-3,7.3$.

${ }^{\mathrm{d}}$ The salt mixture provides the following amounts (g/kg diet $\left.{ }^{-1}\right)$ : Ca, $4 ; \mathrm{K}$, 2.4; Na, 1.6; Mg, 0.4; Fe, 0.12; trace elements: $\mathrm{Mn}, 0.032$; $\mathrm{Cu}, 0.005 ; \mathrm{Zn}$, 0.018; Co, 0.00004; I, 0.00002 .

e The vitamin mixture provides the following amounts ( $\left.\mathrm{mg} / \mathrm{kg} \mathrm{diet}^{-1}\right)$ : retinol, 12; cholecalciferol, 0.125; thiamin, 40; riboflavin, 30; pantothenic acid, 140; pyridoxine, 20; inositol, 300; cyanocobalamine, 0.1; ascorbic acid, 1600; (dL) $\alpha$-tocopherol, 340; menadione, 80; nicotinic acid, 200; paraaminobenzoic acid, 100; folic acid, 10; biotin, 0.6 ; choline, 2720.

\subsection{Preparation of Mitochondria}

At the end of the experimental period and $12 \mathrm{~h}$ after the last feeding, the mice were sacrificed by decapitation and the pancreatic samples were obtained. Mitochondria of pancreas in mice were isolated according to Brustovetsky and Dubinsky [12] with some modifications. Pancreas was moved to preparation buffer including $250 \mathrm{mM}$ sucrose, 1 $\mathrm{mM}$ ethylenediaminetetraacetic acid (EDTA), $20 \mathrm{mM}$ triethanolamine hydrochloride (TRAP), pH 7.4 to isolation. After homogenization, the nuclei and cell debris were separated by centrifugation at $700 \times \mathrm{g}$ for $10 \mathrm{~min}$. Pancreatic mitochondria were obtained after centrifugation of the supernatant at $8000 \times \mathrm{g}$ for $10 \mathrm{~min}$. The mitochondrial pellets were resuspended in ice-cold buffer. All the above procedures were performed at $4^{\circ} \mathrm{C}$. The mitochondrial protein content was determined according to Lowry et al. using bovine serum albumin as standard [13].

\subsection{Assessment of Oxidative Parameters}

\subsubsection{Determination of MDA Content}

Lipid peroxidation was determined by estimating the level of thiobarbituric acid reactive substances. Contents of malondialdehyde (MDA) of isolated mitochondria were measured essentially as described by Koca et al.'s method [14] and the reaction was followed on a spectrophotometer at $532 \mathrm{~nm}$ conditions.

\subsubsection{Determination of NOS Activity}

Nitric oxide synthase (NOS) activity was measured by the NOS assay kit (Biotech Nanjing Chinese built Society) with absorbance of organic layer observed at $530 \mathrm{~nm}$ (Specord 200, Germany) with a spectrophotometer [15]. NOS activity was expressed as unit per gram, one unit/gram meaning $1 \mu$ mol of nitric oxide (NO) formed per gram of protein per minute.

\subsection{Assessment of Antioxidative Parameters}

\subsubsection{Determination of GSH Content}

The non-enzymic antioxidant reduced glutathione (GSH) was analyzed by the method of Tietze [16]. The GSH content was measured using the 5,5'-Dithiobis-(2nitrobenzoic acid) (DTNB) reductase recycling assay for total glutathione, based on reduction of DTNB and formation of a yellow colored anionic product monitored by spectrophotometer at $412 \mathrm{~nm}$.

\subsubsection{Determination of GSH-Px Activity}

Glutathione peroxidase (GSH-Px) activity was measured according to Sabuncu et al. [17]. One unit of GSH-Px was defined as a decrease in the log of mmol GSH per minute and was expressed in unit per mg protein. The automatic decrease of GSH without enzyme (control reaction under same condition) was subtracted from the calculation.

\subsubsection{Determination of SOD Activity}

The activity of antioxidant superoxide dismutase (SOD) was detected by use of the previously reported method of Beauchamp \& Fridovich [18]. The optical density of red product was read at $550 \mathrm{~nm}$. One enzyme unit of SOD activity was defined as the amount of SOD causing $50 \%$ inhibition in $1 \mathrm{mg}$ protein of tissue.

\subsubsection{Determination of CAT Activity}

Colorimetric estimation of catalase (CAT) activity was finished according to the method of Aebi [19]. The principle of the assay is based on the determination of the $\mathrm{H}_{2} \mathrm{O}_{2}$ decomposition rate at $240 \mathrm{~nm}$. One unit of enzyme activity was defined as the amount of enzyme required for monitoring the disappearance of $\mathrm{H}_{2} \mathrm{O}_{2}$ in 1 min.

\subsubsection{Determination of T-AOC Activity}

Total anti-oxidative capacity (T-AOC) was determined according to Koc et al's method [20]. Briefly, potent free radical reactions were initiated with the production of a hydroxyl radical via Fenton reaction and rate of reactions was monitored by following the absorbance of colored dianisidyl radicals. Using this method, antioxidative effect of the sample against potent free radical reactions was measured at 550nm.

\subsection{Statistical Analyses}


SPSS 18.0 statistical software was used for data analysis. Data from different assay treatments were analyzed by one-way analysis of variance (ANOVA); The Student-Neuman-Keuls post-hoc test was used to determine significance for individual experimental conditions. Differences with $P<0.05$ were regarded as significant.

\section{Results}

\subsection{MDA Content and NOS Activity in Pancreatic Mitochondria of Mice}

The content of MDA in pancreatic mitochondria was shown in Figure 1. Compared with the control animals, MDA content in pancreatic mitochondria was significantly increased in STI group $(P<0.05)$. After antioxidant was added to STI diet, MDA content was significanlty decreased $(P<0.05)$, but still higher than that of control group.

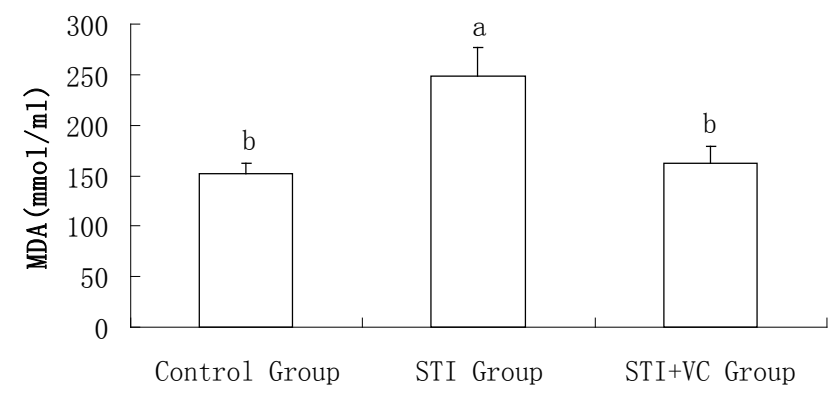

Figure 1. Change of MDA content in pancreatic mitochondria

Figure 2 demonstrated the effect of STI on NOS activity in pancreatic mitochondria. Compared with the control group, NOS activity in pancreatic mitochondria of STI group was significantly increased $(P<0.05)$. The activity of NOS in STI+VC group was significantly lower $(P<0.05)$ than that of STI group, but still higher than that of control group.

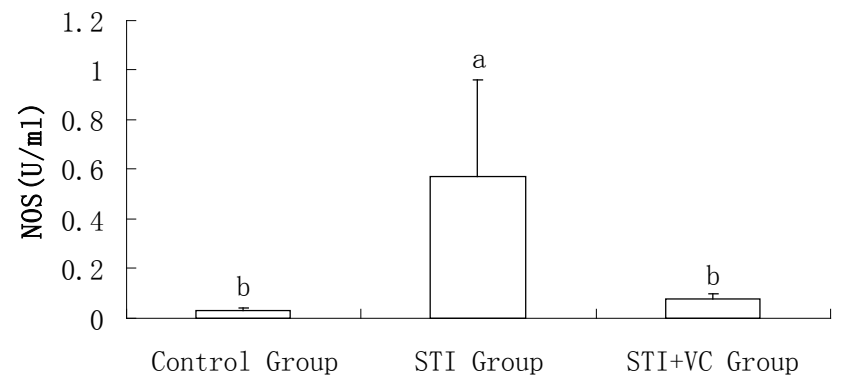

Figure 2. Change of NOS activity in pancreatic mitochondria

\subsection{The levels of Antioxidative Parameters in Pancreatic Mitochondria of Mice}

As shown in Figure 3-Figure 5, compared with the control animals, the activities of enzyme antioxidants (SOD, CAT and GSH-Px) in pancreatic mitochondria were significantly decreased in STI group $(P<0.05)$. The supplement of vitamin $C$ could significantly improve the antioxidative capacity in mitochondria $(P<0.05)$, but activities of SOD, CAT and GSH-Px were still lower than those of control group.

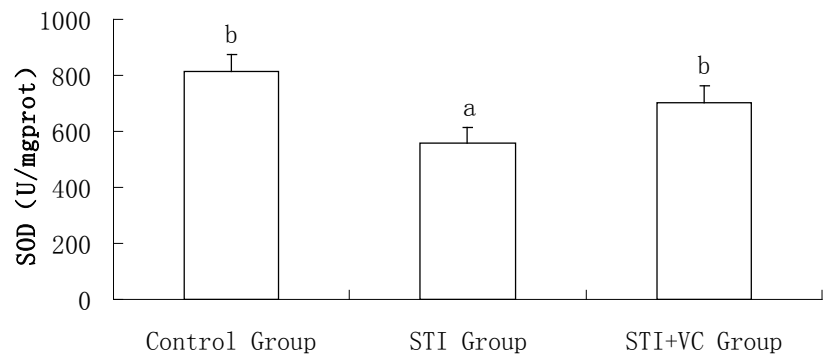

Figure 3. Change of SOD activity in pancreatic mitochondria

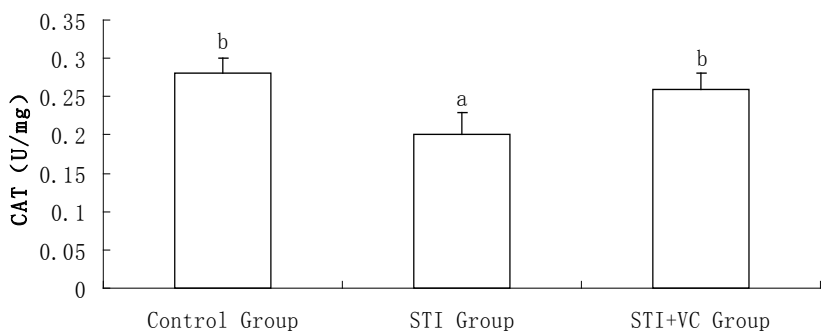

Figure 4. Change of CAT activity in pancreatic mitochondria

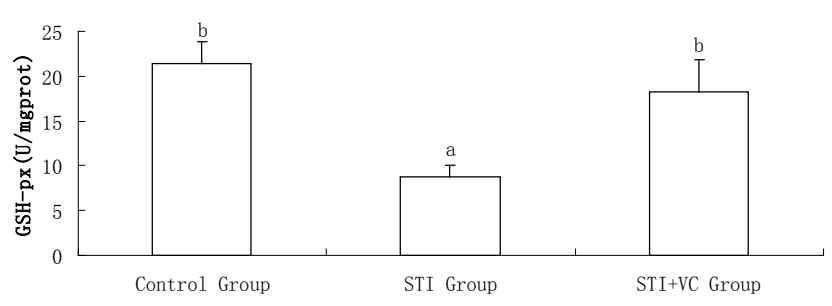

Figure 5. Change of GSH-Px activity in pancreatic mitochondria

Figure 6 showed that, STI could significantly decrease non-enzyme antioxidant, GSH content in pancreatic mitochondria $(P<0.05)$. After vitamin $C$ was supplemented, GSH content was significanlty increased $(P$ $<0.05$ ), but its value was still low compared with that of control group.

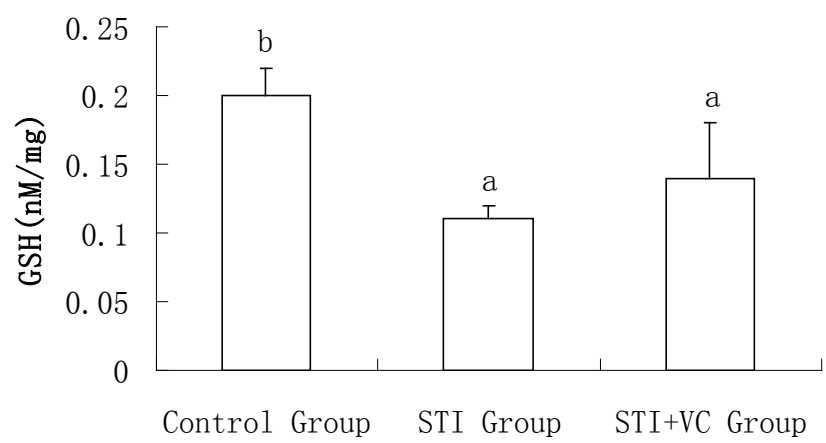

Figure 6. Change of GSH content in pancreatic mitochondria

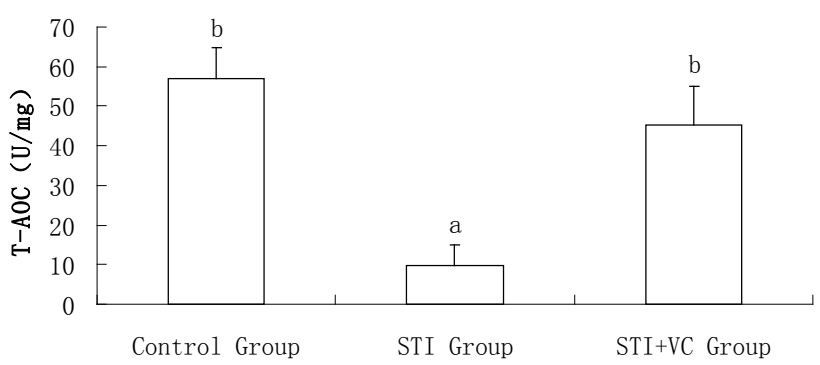

Figure 7. Change of T-AOC activity in pancreatic mitochondria

The result of T-AOC was shown in Figure 7. The comparison among three groups was different and 
statistically significant $(P<0.05)$ except for no significance between control group and STI+VC group.

\section{Discussion}

Mitochondria have been described as "the powerhouses of the cell" because they link the energy-releasing activities of electron transport and proton pumping with the energy conserving process of oxidative phosphorylation, to harness the value of foods in the form of adenosine triphosphate (ATP) [10]. The mitochondrial respiratory chain is followed by production of ROS during energy metabolism [21,22]. If electrons stop flowing through the chain, the proton motive force dissipates and ATP production cannot continue. Nicotinamide adenine denucleotide reduced form (NADH), nicotinamide adenine denucleotide phosphate reduced form (NADPH), and flavin adenine dinucleotide reduced (FADH2) are produced almost exclusively via the aerobic metabolism of protein, fat, and glucose, an increase in dietary energy intake enhances mitochondrial free radical production, which results in oxidative stress [23]. The mitochondrial free radical theory of aging [24] indicated that mitochondria provide most of the energy to the cells, but also are the main source of reactive oxygen species. When rates of free radical production are greater than the scavenging rates, oxidative damage likely occurs in cells and tissues. It is reported that redox imbalance between plasma and liver mitochondria might become a major threat to chronic diabetic rats [25]. Early study showed that doxorubicin causes significant cardiotoxicity characterized increases in oxidative stress and mitochondrial dysfunction [26]. It is reported that malathion has been shown to induce oxidative stress, lead to the lipid peroxidation of brain mitochondria [27].

Lipid peroxidation has been suggested to be a main mechanism of oxidative stress [28]. MDA is a product of multiple unsaturated fatty acid peroxidation, often used as a reliable marker of lipid peroxidation [29]. In our investigation, MDA content in pancreatic mitochondria of mice fed STI diet was significantly increased $(P<0.05)$, which demonstrated that lipid peroxidation were increased by STI. Similar results were observed in naphthalenetreated rats, free radicals levels of mitochondria in liver and kidney were increased, lipid peroxidation was increased and glutathione content was decreased [30].

NOS produces NO by catalyzing the conversion of Larginine to Lcitrulline, with the concomitant oxidation of NADPH, which have slow down the velocity of the electrons in the respiratory chain to inhibiting the breath and reducing the consumption of oxygen, causing a state of stress leading to mitochondrial lipid peroxidation [31,32]. In our study, NOS activities in mitochondria of STI group were significantly increased $(P<0.05)$. Prasenjit Manna et al. also found that hyperglycemia increased production of ROS, increased lipid peroxidation, and increased activity of inducible nitric oxide synthase (iNOS) [33]. All above results demonstrated that STI can lead to the increase of free radicals levels in pancreatic mitochondria.

The main antioxidant defense system includes enzymatic reaction system and non enzymatic reaction system, enzymatic system mainly for SOD, CAT and
GSH-Px; non enzymatic system mainly for vitamins ( vitamin C, vitamin E etc.), amino acid and so on [34]. SOD molecules mediate scavenging of ROS to $\mathrm{H}_{2} \mathrm{O}_{2}$ and molecular oxygen. They belong to a large family of isoenzymes that mediate cellular response to oxidative stress and represent the main enzymatic source of peroxides [35,36]. CAT is an important antioxidant enzyme that protects life against harmful effects of $\mathrm{H}_{2} \mathrm{O}_{2}$ through disproportionating it into molecular oxygen and water [37]. GSH-Px by reduction of GSH to catalyze the hydrogen peroxide and organic peroxides, is an important component of antioxidant system [38]. Our results showed that activities of SOD, CAT and GSH-Px in STI group were significantly decreased compared with those of the control animals. Similar results could be observed that SOD activity in pancreatic mitochondria of diabetes was decreased by oxidative stress [39]. Hilaire Bakala et al. found that the key role of mitochondrial catalase of rat liver in antioxidant action was recently demonstrated by decreasing catalase activity [40]. It is reported that the activities of SOD and GSH-PX were decreased by endosulfan-induced oxidative stress, which lead to mitochondrial energy metabolism dysfunction involved in reproductive toxicity of mice [41]. These results suggested that the excessive free radicals induced by STI consumed a large number of antioxidant and caused the antioxidative capacity weakened in organism.

GSH is the important part of non enzymatic antioxidants. GSH can remove peroxynitrite (ONOO-) with the formation of oxidized glutathione (GS-SG), which is converted back into GSH by the NADPHdependent glutathione reductase [42]. T-AOC can eliminate free radicals and reactive oxygen species (O-2), blocking peroxidation chain, to avoid the occurrence of lipid peroxidation and can remove the catalytic metal ion [43]. It is reported that in heart mitochondria the T. cruziinduced oxidative stress occurred by activation of glutathione antioxidant defenses to control the oxidative damage which decreased the GSH content of mice [44]. Samir Mandal et al. found that enhanced hepatotoxicity might result in oxidative stress in liver, and GSH content was decreased in antioxidant defense system [45]. In our study, it was found that GSH content and T-AOC level in STI group were significantly decreased compared with those of the control animals $(P<0.05)$. Taken together, these results suggested that excessive production of free radicals in pancreatic mitochondria consumed a large number of enzymatic antioxidants and non enzymatic antioxidants so these antioxidants were significantly decreased, which is consistent with the results of previous reports $[40,41]$.

Vitamin C (ascorbic acid), as a very powerful antioxidant agent, can act both, directly, via scavenging of reactive oxygen species, and indirectly, through regeneration of other antioxidant systems [46]. This vitamin was shown to protect effect against free radicalinduced oxidative damage [47]. In our investigation, vitamin C could reduce excessive production of free radicals caused by STI, thus protect the body from the damage of endogenous oxygen free radical.

\section{Conclusions}


In summary, the results of our study suggested that soybean trypsin inhibitor exhibited harmful effect on pancreatic mitochondria, which induced oxidative stress by increasing the formation of lipid peroxidation and overall impairing enzymatic and nonenzymatic antioxidant defenses in mice. Treatment with supplement vitamin $C$ in soybean trypsin inhibitor diet significantly reduced extent of oxidative stress in pancreatic mitochondria of mice, which further demonstrated that soybean trypsin inhibitor may destroy the balance of oxidant and antioxidant in organism by inducing generation of free radicals.

\section{Acknowledgments}

The authors want to thank National Natural Science Foundation of China (NSFC, No.31000769), Postdoctoral Library of Jilin Agricultural University and Project funded by China Postdoctoral Sciemce Foundation (Grant, No.2012M520690) for the funding.

\section{References}

[1] Roychaudhuri R, Sarath G, Zeece M, Markwell J, "Stability of the allergenic soybean Kunitz trypsin inhibitor". Biochimica et Biophysica Acta (BBA) - Proteins and Proteomics 1699, 207-212, 2004.

[2] Jin B, Tian SJ, "Review on soybean trypsin inhibitor". Journal of Cereal Oils 6: 3-5, 2005.

[3] Baintner K, "Trypsin inhibitor and chymotrypsin inhibitor studies with soybean extracts". Journal of Agricultural and Food Chemistry 29, 201-205, 1981.

[4] Bau HM, Villaume C, Giannangeli F, Nicolas JP, Mejean L, "Optimisation du chauffage et valeurs nutritionnelle et fonctionnelle des prote 'ines de soja". Cahiers de nutrition et de die'te 'tique 36, 96-102, 2001.

[5] Schulze H, Huisman J, Verstegen MWA, et al, "Physiological effects of isolated soya trypsin inhibitors (STI) on pigs. In: van der Poel AFB, Huisman J, Saimi HS, ed. Recent Advances of Research in Antinutritional Factors in Legume Seeds: Wageningen Pers, Wageningen [M]”. The NETH 195-9, 1993.

[6] Herkelman KL, Cromwell GL, Stahly TS, et al, "Apparent digestibility of amino acids in raw and heated conventional and low trypsin inhibitor soybean for pigs". Journal of Animal Science 70, 818-26, 1992.

[7] Struthers BJ, McDonald JR, "Effects of raw soy flour feeding in weaning pigs: comparison with rats and monkeys". Qualitas Plant Plant Foods Human Nutrition 35, 331-8, 1985.

[8] Li SF, Yang LJ, Huo GC, Feng MS, Ma JF, "Study on the Tolerance of Broilers to Dietary Trypsin Inhibitor". China Poultry 3, 8-10, 2000.

[9] Paradies G, Petrosillo G, Paradies V, Ruggiero FM, "Oxidative stress, mitochondrial bioenergetics, and cardiolipin in aging”. Free radical biology and Medicine 48, 1286-1295, 2010.

[10] Cadenas E, Davies KJ, "Mitochondrial Free Radical Generation, oxidative stress, and aging”. Free radical biology and Medicine 29, 222-230, 2000.

[11] Gu CM, Han LL, Zhao LL, et al, "The effect of soybean trypsin inhibitor on generation of oxygen free radical in vivo at different growth stages of mice”. Acta Veterinaria et Zootechnica Sinica 44, 1425-1431, 2013.

[12] Brustovetsky N, Dubinsky JM, "Limitations of cyclosporin A inhibition of the permeability transition in CNS mitochondria". Journal of Neuroscience 20, 8229-8237, 2000.

[13] Lowry OH, Rosebrough NJ, Farr AL, Randall RJ, "Protein measurement with the folin phenol reagent”. Biological Chemistry 193, 265-275, 1951.

[14] Koca K, Yurttas Y, Bilgic S, Cayci T, Topal T, Durusu M, et al, "Effect of preconditioned hyperbaric oxygen and ozone on ischemia-reperfusion induced tourniquet in skeletal bone of rats". Journal of Surgical Research 164, e83-e89, 2010.
[15] Knowles RG, Salter M, Brooks SL, "Anti-inflammatory glucocirti-coids inhibit the induction by endotoxin of nitric oxide synthase in the lung, liver and aorta of the rat". Biochemical and Biophysical Research Communications 172, 1042-8, 1990.

[16] Tietze F, "Enzymic method for quantitative determination of nanogram amounts of total and oxidized glutathione: applications to mammalian blood and other tissues". Analytical Biochemistry 27, 502-522, 1969.

[17] Sabuncu T, Vural H, Harma M, Harma M, “Oxidative stress in polycystic ovary syndrome and its contribution to the risk of cardiovascular disease”. Clinical Biochemistry 34, 407-413, 2001.

[18] Beauchamp C, Fridovich I, "Superoxide dismutase: Improved assays and an assay applicable to acrylamide gels”. Analytical Biochemistry 44, 276-287, 1971.

[19] Aebi H, “Catalase in vitro". Methods Enzymol 105, 121-126, 1984

[20] Koc S, Aksoy N, Bilinc H, Duygu F, Uysal IÖ, Ekinci A, "Paraoxonase and arylesterase activity and total oxidative/antioxidative status in patients with chronic adenotonsillitis". International journal of Pediatric Otorhinolaryngology 75, 13641367, 2011.

[21] Chance B, Sies H, Boveris A, "Hydroperoxide metabolism in mammalian organs”. Physiological Reviews 59, 527-605, 1979.

[22] Murphy MP, "How mitochondria produce reactive oxygen species”. Biochemical Journal 417, 1-13, 2009.

[23] Freidovich I, "Fundamental aspects of reactive oxygen species, or what's the matter with oxygen?" Annals of the New York Academy of Sciences 893, 13, 1999.

[24] Harman D, “The biologic clock: the mitochondria?” Journal of the American Geriatrics Society 20, 145-147, 1972.

[25] Cakatay U, Kayali R, "The evaluation of altered redox status in plasma and mitochondria of acute and chronic diabetic rats". Clinical Biochemistry 39, 907-912, 2006.

[26] Granados-Principal S, El-azem N, Pamplona R, Ramirez-Tortosa $\mathrm{C}$, et al, "Hydroxytyrosol ameliorates oxidative stress and mitochondrial dysfunction in doxorubicin-induced cardiotoxicity in rats with breast cancer”. Biochemical Pharmacology, in press.

[27] Rezvanfar MA, Rezvanfar MA, Ranjbar A, Baeeri M, Mohammadirad A, Abdollahi M, "Biochemical evidence on positive effects of rolipram a phosphodiesterase-4 inhibitor in malathion-induced toxic stress in rat blood and brain mitochondria”. Pesticide Biochemistry and Physiology 98, 135143, 2010.

[28] Fang YZ, Yang S, Wu GY, "Free Radicals, Antioxidants, and Nutrition”. Nutrition 18, 872- 879, 2002.

[29] Fang YZ. Free radicals and nutrition. In: Fang YZ, Zheng RL, eds, "Theory and application of free radical biology". Beijing: Scientific Press 647, 2002.

[30] Mantawy EM, El-Bakly WM, Esmat A, Badr AM, El-Demerdash E, "Chrysin alleviates acute doxorubicin cardiotoxicity in rats via suppression of oxidative stress, inflammation and apoptosis". European Journal of Pharmacology 728, 107-118, 2014.

[31] Brown GC, Borutaite V, "Nitric oxide and mitochondrial respiration in the heart”. Cardiovascular Research 75, 283-290, 2007.

[32] Solien J, Haynes V, Giulivi C, "Differential requirements of calcium for oxoglutarate dehydrogenase and mitochondrial nitricoxide synthase under hypoxia: impact on the regulation of mitochondrial oxygen consumption”. comparative biochemistry and physiology a-molecular \& integrative physiolog 142, 111-117, 2005.

[33] Fischer-Nielsen A, Poulsen HE, Loft S, “8Hydroxydeoxyguanosine in vitro: effects of glutathione, ascorbate, and 5-aminosalicylic acid”. Free Radical Biology and Medicine 13, 121, 1992.

[34] Qin SZ, Yu QF, Ma GX, Zhou Z, Hao WW, Li MG, "Effect of Combined Stress on Plasma CuZn-SOD and Erythrocyte Membrane $\mathrm{T}$ AOC in Pilots during Low Altitude Flight in Summer". Space Medicine \& Medical Engineering-Academic work 13 (3), 2000.

[35] Miller AF, "Superoxide dismutases: ancient enzymes and new insights”. febs letters 586, 585-595, 2012.

[36] Mishra P, Dixit A, Ray M, Sabat SC, "Mechanistic study of CuZnSOD fromIpomoea carnea mutated at dimer interface: Enhancement of peroxidase activity upon monomerization". Biochimie 97, 181-193, 2014.

[37] Bakala H, Hamelin M, Mary J, Borot-Laloi C, Friguet B, "Catalase, a target of glycation damage in rat liver mitochondria with aging”. Biochim Biophys Acta 1822, 1527-1534, 2012. 
[38] Lauterberg BH, Corcoran GB, hlitchell JR, "Mechanism of action of $\mathrm{N}$-acetylcysteine in the protection against the hepatotoxicity of acetaminophen in rats in vlvo". Journal of Clinical Investigation 71, 980-991, 1983.

[39] Karatug A, Bolken S, "The potential role of combined antioxidant treatment on pancreas of STZ-diabetic mice". Experimental and Toxicologic Pathology 65, 255-262, 2013.

[40] Tehrani HS, Moosavi-Movahedi AA, Ghourchian H, "Correlation between biological activity and electron transferring of bovine liver catalase: Osmolytes effects”. Electrochimica Acta 113, 591602, 2013.

[41] Bhosle SM, Huilgol NG, Mishra KP, "Enhancement of radiationinduced oxidative stress and cytotoxicityin tumor cells by ellagic acid”. Clinica Chimica Acta 359, 89-100, 2005.

[42] Sies H, "Glutathione and its role in cellular functions". Free Radical Biology and Medicine 27, 916, 1999.

[43] Yang J, Gao F, "The Research of The ROS and The Apoptosis". Foreign Medical Sciences Cancer Section 29, 248, 2002.
[44] Wen JJ, Vyatkina G, Garg N, “Oxidative damage during chagasic cardiomyopathy development: role of mitochondrial oxidant release and inefficient antioxidant defense". Free Radical Biology and Medicine 37, 1821-1833, 2004.

[45] Mandal S, Nelson VK, Mukhopadhyay S, Bandhopadhyay S, Maganti L, Ghoshal N, Sen G, Biswas T, "14Deoxyandrographolide targets adenylate cyclase and prevents ethanol-induced liver injury through constitutive NOS dependent reduced redox signaling in rats”. Food and Chemical Toxicology 59, 236-248, 2013.

[46] Manna P, Das J, Ghosh J, Sil PC, “Contribution of type 1 diabetes to rat liver dysfunction and cellular damage via activation of NOS, PARP, I $\mathrm{KB} \alpha / \mathrm{NF}-\kappa \mathrm{B}, \mathrm{MAPKs}$, and mitochondria-dependent pathways: Prophylactic role of arjunolic acid”. Free Radical Biology and Medicine 48, 1465-1484, 2010.

[47] Kontek R, Jakubczak M, Matlawska-Wasowska K, "The antioxidants, vitamin $\mathrm{A}$ and $\mathrm{E}$ but not vitamin $\mathrm{C}$ and melatonin enhance the proapoptotic effects of irinotecan in cancer cellsin vitro”. Toxicology in Vitro 28, 282-291, 2014. 